\section{Col 03}

PREVALENCE AND TOPOGRAPHICAL DISTRIBUTION OF COLORECTAL ADENOMAS - A STUDY ON 1006 AUTOPSIES.

H. Gabbert, C.-D. Gerharz, R. Wagrier

The increasing frequency of carcinomas in the proximal colon ("shift to the right") has also turned up new questions concerning the prevalence and localization of the colorectal adenomas. Based on 1006 unselected autopsies including 199 adenoma patients with a total of 498 adenomas - we could get the following results: 1.) The percentage of adenoma patients increases continuously from $6 \%$ in the age group of the $40-50$ year old patients to $38.5 \%$ in the age group of the $90-100$ year old patients. No adenomas were present in the age group of patients under 40 years. 2.) Only $6 \%$ respectively $8 \%$ of all adenomas were localized in the coecum and rectum, whereas all the other adenomas were rather eveniy distributed over the c.ascendens (23\%), c.transversum (25\%), c.descendens (15 s) and c.sigmoideum (23 \%). 3.) When analyzing the topographical distribution of the adenomas separately for the individual age groups an age-dependent shift to the right can be shown in the proximal parts of the large intestine where the percentage of the adenomas increases with age, whereas the opposite is true for the distal part of the large intestine. 4.) As shown by comparison of the absolute numer of adenomas, this shift to the right is based on an overproportional increase of the adenoma number in the proximal colon in the old age when compared with the distal parts of the colon. 5.) This increasing predilection for the proximal colon in the old age is also accompanied by a change of the adenoma density (number of adenomas per mucosal square unit) which in the $40-60$ year old patients increases continuously from the coecum to the rectum, whereas the opposite is true for the 80 100 year old patients. This means, that the adenoma risk per mucosal square unit is in the young age highest in the rectum and in the old age highest in the coecum.

Pathologisches Institut der Universität, Langenbeckstr. 1, D- 6500 Mainz

\section{Col 04}

DO BIOPSIES OF COLORECTAL CARCINOMAS ALLOW FOR AN ADEQUATE GRADING?

\section{K. Donhuijsen, J. Mann and L.-D. Leder}

To answer this question, in 100 cases, a panel of histologic criteria was analysed. Furthermore, the grade of malignancy of each case was assessed by two independent investigators, both with the biopsies and the surgical specimens.

It was found that there is a marked inhomogeneity of both histological and cytological parameters within an individual carcinoma and also from case to case. However, on their surfaces, the tumors exhibited often adenopapillary formations whereas deeper areas were often solid and undifferentiated.

Since carcinomas of the large bowel, at their surfaces, usually tend to show lower grades of malignancy, the value of grading of colorectal carcinomas with biopsies alone will not render reliable results, which diminishes the value of such assessments of biopsies alone considerably. The same could be found by flow cytometric investigations (Petersen et al. 7980). Therefore, we conclude that a sufficient and valid characterization of the biology of colorectal carcinomas is not possible with preoperatively obtained biopsy materials.

Universitätsinstitut für Pathologie, Westdeutsches Tumorzentrum, Hufelandstr. 55, D- 4300 Essen 1

\section{Col 05}

ULTRASTRUCTURAL DIFFERENTIATION IN COLORECTAL CARCINOMA H.P. Sinn (a.G.), H.F. Kern

Light microscopic grading in colonectal carcinoma has been debatable, because objective criteria have not been established. The WHO proposes to place equal weight on structural and on cytological criteria. We asked, if the light microscopical degree of differentiation can be verified at the ultrastructural level, and if so, which light microscopic criteria are valid.

We examined 16 colorectal adenocarcinomas (excluding mucinous adenocarcinomas) and compared light microscopic criteria with the ultrastructural criteria of differentiation. Light microscopical grading was performed on giant histologic sections, representative areas identified on semithin sections and processed for electron microscopy. At least three ultrathin sections were examined per case.

UItrastructurally we identified seven different cell types in colorectal adenocarcinoma: undifferentiated cell (UC), intemediate cell (IC), immature goblet cell (IG), immature absorptive cell (IA), mature goblet cel I (MG), mature absorptive cell (MA) and enterochromaff in cell (EC). These cell types were present in variable amounts in light microscopically well, moderately and poorly differentiated carcinomas. There was a good correlation between the light microscopic and the electron microscopic grading, when the light microscopic grading was based on three criteria: cell polarity, nuclear atypia and stratification. Other light microscopical criteria, such as glandular arrangement, budding, branching, bridging and papil lary fomations were of less predictive value in view of the degree of ultrastructural cytoplasmatic differentiation.

Considering the limited number of cases examined and the limited number of ultrathin sections available, these results must be looked upon with caution. One firm conclusion can still be drawn: The light microscopic grading is a good indicator of the number of immature and undifferentiated cells at the ultrastructural level. More emphasis should be placed on cytologic than on structural criteria.

Medizinisches Zentrum für Pathologie der Philipos-Universität, Klinikum Lahnberge, Conradistraße, D-3550 Marburg.

Institut für Anatomie und Zellbiologie der Philipps-Universität, Robert-Koch-Straße 8, D-3550 Marburg

\section{Col 06}

SIMULTANEOUSNESS OBSERVATION OF GOBLET CELL CARCINOID AND DIVERTICULA OF THE APPENDIX. HISTOLOGICAL, IMMUNHISTOCHEMICAL AND ELECTRONMICROSCOPICAL INVESTIGATIONS.

\section{J. Vogel, V. Totovic, A. Beck, P. Gedigk}

Carcinoids belong to the most common of all tumors of the appendix. A coincidence of a carcinoid tumor and diverticula with in the appendix wall could not be found in the extensively searched literatur. In a 57 old female an acute inflamed appendix with a small perforation could be found intraoperatively. The histopathology of the appendix revealed beside an ulcerative and suppurative appendicitis also an inflammatory reaction of the mesenteriolar fatty tissue. In addition there were multiple outpouchings of the appendiceal wall and of the mucosa in form of true and false Diverticula. In the wall of one diverticulum as well as in the tissue surrounding a false diverticulum there were diffuse formations of a goblet cell carcinoid infiltrating the entire thickness of the wall of the appendix. The histological, immunhistochemical and electronmicroscopical workup revealed an argentaffinic and an argyrophilic goblet cell carcinoid with proof of characteristic mucin - and enterochromaffinic granules as well as with positive reactions for PAS, CEA and TPA. Muco-argentaffinic carcinoids occure in the appendix, colon and in the duodenum only rarely. Also infiltration of the mucosa has been described only occasionally. In addition, the infiltration of the appendiceal wall, perineural invasion and infiltration of the mesenteriolar fatty tissue are rarities. Usually the mucosal lining is not ulcerated. This observed case, however, presented with symptoms of an acute appendicitis due to ulcerations and perforation which were caused by inflamed diverticula.

Pathologisches Institut der Universität, Sigmund-FreudStr. 25, 5300 Bonn 1 (Venusberg) 